\title{
Variational approach to a class of impulsive differential equations
}

Dajun Guo*

\section{"Correspondence:} guodj@sdu.edu.cn

Department of Mathematics, Shandong University, Jinan, Shandong 250100, People's Republic of China

\begin{abstract}
In this article, the author discusses the existence of solutions for a class of impulsive differential equations by means of a variational approach different from earlier approaches.

MSC: 34B37; 45G10;47H30; 47J30

Keywords: impulsive differential equation; integral equation; variational method; critical point theory
\end{abstract}

\section{Introduction}

The theory of impulsive differential equations has been emerging as an important area of investigation in recent years [1-3]. There is a vast literature on the existence of solutions by using topological methods, including fixed point theorems, Leray-Schauder degree theory, and fixed point index theory [4-15]. But it is quite difficult to apply the variational approach to an impulsive differential equation; therefore, there was no result in this area for a long time. Only in the recent five years, there appeared a few articles which dealt with some impulsive differential equations by using variational methods [16-20]. Motivated by [17], in this article we shall use a different variational approach to discuss the existence of solutions for a class of impulsive differential equations and we only deal with classical solutions.

Consider the boundary value problem (BVP) for the second-order nonlinear impulsive differential equation:

$$
\left\{\begin{array}{l}
-u^{\prime \prime}(t)=f(t, u(t)), \quad \forall t \in J^{\prime}, \\
\left.\Delta u\right|_{t=t_{k}}=c_{k} \quad(k=1,2,3, \ldots, m), \\
\left.\Delta u^{\prime}\right|_{t=t_{k}}=d_{k} \quad(k=1,2,3, \ldots, m), \\
u(0)=u(1)=0
\end{array}\right.
$$

where $J=[0,1], 0<t_{1}<\cdots<t_{k}<\cdots<t_{m}<1, J^{\prime}=J \backslash\left\{t_{1}, \ldots, t_{k}, \ldots, t_{m}\right\}, c_{k}$ and $d_{k}(k=$ $1,2, \ldots, m)$ are any real numbers, $f(t, u)$ is a real function defined on $J \times R$, where $R$ denotes the set of all real numbers, and $f(t, u)$ is continuous on $J^{\prime} \times R$, left continuous at $t=t_{k}$, i.e.

$$
\lim _{t \rightarrow t_{k}-0, w \rightarrow u} f(t, w)=f\left(t_{k}, u\right)
$$

(02014 Guo; licensee Springer. This is an Open Access article distributed under the terms of the Creative Commons Attribution License (http://creativecommons.org/licenses/by/2.0), which permits unrestricted use, distribution, and reproduction in any medium, provided the original work is properly cited. 
for any $u \in R(k=1,2, \ldots, m)$, and the right limit at $t=t_{k}$ exists, i.e.

$$
\lim _{t \rightarrow t_{k}+0, w \rightarrow u} f(t, w)
$$

(denoted by $f\left(t_{k}^{+}, u\right)$ ) exists for any $u \in R(k=1,2, \ldots, m) .\left.\Delta u\right|_{t=t_{k}}$ denotes the jump of $u(t)$ at $t=t_{k}$, i.e.

$$
\left.\Delta u\right|_{t=t_{k}}=u\left(t_{k}^{+}\right)-u\left(t_{k}^{-}\right)
$$

where $u\left(t_{k}^{+}\right)$and $u\left(t_{k}^{-}\right)$represent the right and left limits of $u(t)$ at $t=t_{k}$, respectively. Similarly,

$$
\left.\Delta u^{\prime}\right|_{t=t_{k}}=u^{\prime}\left(t_{k}^{+}\right)-u^{\prime}\left(t_{k}^{-}\right),
$$

where $u^{\prime}\left(t_{k}^{+}\right)$and $u^{\prime}\left(t_{k}^{-}\right)$represent the right and left limits of $u^{\prime}(t)$ at $t=t_{k}$, respectively. Let $P C[J, R]=\left\{u: u\right.$ is a real function on $J$ such that $u(t)$ is continuous at $t \neq t_{k}$, left continuous at $t=t_{k}$, and $u\left(t_{k}^{+}\right)$exists, $\left.k=1,2, \ldots, m\right\}$ and $P C^{1}[J, R]=\left\{u \in P C[J, R]: u^{\prime}(t)\right.$ is continuous at $t \neq t_{k}$ and $u^{\prime}\left(t_{k}^{+}\right), u^{\prime}\left(t_{k}^{-}\right)$exist, $\left.k=1,2, \ldots, m\right\}$. A function $u \in P C^{1}[J, R] \cap C^{2}\left[J^{\prime}, R\right]$ is called a solution of BVP (1) if $u(t)$ satisfies (1).

Let us list some conditions.

$\left(\mathrm{H}_{1}\right)$ There exist $p>2, a>0$ and $b>0$ such that

$$
|f(t, u)| \leq a+b|u|^{p-1}, \quad \forall t \in J, u \in R
$$

$\left(\mathrm{H}_{2}\right)$ There exist $0<c<\frac{\pi^{2}}{4}$ and $d>0$ such that

$$
\int_{0}^{u} f(t, v) d v \leq c u^{2}+d, \quad \forall t \in J, u \in R
$$

Lemma $1 u \in P C^{1}[J, R] \cap C^{2}\left[J^{\prime}, R\right]$ is a solution of $B V P$ (1) if and only if $v \in C[, R]$ is a solution of the integral equation

$$
v(t)=\int_{0}^{1} G(t, s) g(s, v(s)) d s, \quad \forall t \in J
$$

where

$$
\begin{aligned}
& G(t, s)= \begin{cases}s(1-t), \quad \forall 0 \leq s \leq t \leq 1 ; \\
t(1-s), & \forall 0 \leq t<s \leq 1,\end{cases} \\
& g(t, v)=f(t, v+a(t)-a(1) t), \quad \forall t \in J, v \in R
\end{aligned}
$$

and

$$
v(t)=u(t)-a(t)+a(1) t, \quad a(t)=\sum_{0<t_{k}<t}\left[c_{k}+\left(t-t_{k}\right) d_{k}\right], \quad \forall t \in J .
$$


Proof For $u \in P C^{1}[J, R] \cap C^{2}\left[J^{\prime}, R\right]$, we have the formula (see [21], Lemma 1(b))

$$
\begin{aligned}
u(t)= & u(0)+t u^{\prime}(0)+\int_{0}^{t}(t-s) u^{\prime \prime}(s) d s \\
& +\sum_{0<t_{k}<t}\left\{\left[u\left(t_{k}^{+}\right)-u\left(t_{k}\right)\right]+\left(t-t_{k}\right)\left[u^{\prime}\left(t_{k}^{+}\right)-u^{\prime}\left(t_{k}^{-}\right)\right]\right\}, \quad \forall t \in J .
\end{aligned}
$$

So, if $u \in P C^{1}[J, R] \cap C^{2}\left[J^{\prime}, R\right]$ is a solution of BVP (1), then, by (1) and (6), we have

$$
\begin{aligned}
u(t) & =t u^{\prime}(0)-\int_{0}^{t}(t-s) f(s, u(s)) d s+\sum_{0<t_{k}<t}\left[c_{k}+\left(t-t_{k}\right) d_{k}\right] \\
& =t u^{\prime}(0)-\int_{0}^{t}(t-s) f(s, u(s)) d s+a(t), \quad \forall t \in J .
\end{aligned}
$$

It is clear, by (5), that

$$
a(t)=0, \quad \forall 0 \leq t \leq t_{1} ; \quad a(1)=\sum_{k=1}^{m}\left[c_{k}+\left(1-t_{k}\right) d_{k}\right]
$$

so

$$
v^{\prime}(0)=u^{\prime}(0)+a(1) .
$$

Substituting (9) into (7), we get

$$
\begin{aligned}
v(t) & =t v^{\prime}(0)-\int_{0}^{t}(t-s) f(s, u(s)) d s \\
& =t v^{\prime}(0)-\int_{0}^{t}(t-s) f(s, v(s)+a(s)-a(1) s) d s \\
& =t v^{\prime}(0)-\int_{0}^{t}(t-s) g(s, v(s)) d s, \quad \forall t \in J .
\end{aligned}
$$

By virtue of (5), we see that $v \in C[J, R]$ (in fact, $v \in C^{1}[J, R]$ ) and

$$
v(1)=u(1)-a(1)+a(1)=u(1)=0,
$$

so, letting $t=1$ in (10), we find

$$
v^{\prime}(0)=\int_{0}^{1}(1-s) g(s, v(s)) d s .
$$

Substituting (11) into (10), we get

$$
\begin{aligned}
v(t) & =\int_{t}^{1} t(1-s) g(s, v(s)) d s+\int_{0}^{t} s(1-t) g(s, v(s)) d s \\
& =\int_{0}^{1} G(t, s) g(s, v(s)) d s, \quad \forall t \in J,
\end{aligned}
$$

so $v(t)$ is a solution of the integral equation (2). 
Conversely, suppose that $v \in C[J, R]$ is a solution of (2), i.e.

$$
v(t)=(1-t) \int_{0}^{t} s g(s, v(s)) d s+t \int_{t}^{1}(1-s) g(s, v(s)) d s, \quad \forall t \in J .
$$

By (4), it is clear that $g(t, v(t))$ is continuous on $J^{\prime}$, so differentiation of (12) gives

$$
\begin{aligned}
v^{\prime}(t)= & -\int_{0}^{t} s g(s, v(s)) d s+(1-t) \operatorname{tg}(t, v(t)) \\
& +\int_{t}^{1}(1-s) g(s, v(s)) d s-t(1-t) g(t, v(t)) \\
= & -\int_{0}^{t} s g(s, v(s)) d s+\int_{t}^{1}(1-s) g(s, v(s)) d s, \quad \forall t \in J^{\prime} .
\end{aligned}
$$

Differentiating again, we get

$$
v^{\prime \prime}(t)=-\operatorname{tg}(t, v(t))-(1-t) g(t, v(t))=-g(t, v(t)), \quad \forall t \in J^{\prime} .
$$

From $(13)$ we see that $v^{\prime}\left(t_{k}^{+}\right)$and $v^{\prime}\left(t_{k}^{-}\right)(k=1,2, \ldots, m)$ exist and

$$
v^{\prime}\left(t_{k}^{+}\right)=v^{\prime}\left(t_{k}^{-}\right)=-\int_{0}^{t_{k}} s g(s, v(s)) d s+\int_{t_{k}}^{1}(1-s) g(s, v(s)) d s .
$$

It follows from (4), (5), (12), (14), and (15) that $u \in P C^{1}[J, R] \cap C^{2}\left[J^{\prime}, R\right]$ and $u(t)$ satisfies (1).

Lemma 2 Let condition $\left(\mathrm{H}_{1}\right)$ be satisfied. If $v \in L^{p}[J, R]$ is a solution of the integral equation (2), then $v \in C[J, R]$.

Proof It is clear, for function $a(t)$ defined by (5),

$$
|a(t)| \leq a_{0}, \quad \forall t \in J ; \quad a_{0}=\sum_{k=1}^{m}\left(\left|c_{k}\right|+\left(1-t_{k}\right)\left|d_{k}\right|\right)
$$

By (4), (5), (16), and condition $\left(\mathrm{H}_{1}\right)$, we have

$$
\begin{aligned}
|g(t, v)| & \leq a+b|v+a(t)-a(1) t|^{p-1} \leq a+b\left(|v|+2 a_{0}\right)^{p-1} \\
& \leq a+b\left(2 \max \left\{|v|, 2 a_{0}\right\}\right)^{p-1} \leq a+b 2^{p-1}\left(|v|^{p-1}+\left(2 a_{0}\right)^{p-1}\right), \quad \forall t \in J, v \in R,
\end{aligned}
$$

so,

$$
|g(t, v)| \leq a_{1}+b_{1}|v|^{p-1}, \quad \forall t \in J, v \in R
$$

where

$$
a_{1}=a+b 2^{2(p-1)} a_{0}^{p-1}, \quad b_{1}=b 2^{p-1} .
$$


It is clear that $g(t, v)$ satisfies the Caratheodory condition, i.e. $g(t, v)$ is measurable with respect to $t$ on $J$ for every $v \in R$ and is continuous with respect to $v$ on $R$ for almost $t \in J$ (in fact, $g(t, v)$ is discontinuous only at $t=t_{k}(k=1,2, \ldots, m)$ ), so (17) implies [22, 23] that the operator $g$ defined by

$$
(g v)(t)=g(t, v(t)), \quad \forall t \in J
$$

is bounded and continuous from $L^{p}[J, R]$ into $L^{q}[J, R]$, where $\frac{1}{p}+\frac{1}{q}=1(q>1)$.

Let $v \in L^{p}[J, R]$ be a solution of the integral equation (2). Then by the Hölder inequality,

$$
\left|v\left(t_{1}\right)-v\left(t_{2}\right)\right| \leq\left(\int_{0}^{1}\left|G\left(t_{1}, s\right)-G\left(t_{2}, s\right)\right|^{p} d s\right)^{\frac{1}{p}}\left(\int_{0}^{1}|g(s, v(s))|^{q} d s\right)^{\frac{1}{q}}, \quad \forall t_{1}, t_{2} \in J,
$$

which implies by virtue of the uniform continuity of $G(t, s)$ on $J \times J$ that $v \in C[, R]$.

\section{Variational approach}

Theorem 1 If conditions $\left(\mathrm{H}_{1}\right)$ and $\left(\mathrm{H}_{2}\right)$ are satisfied, then $B V P(1)$ has at least one solution $u \in P C^{1}[J, R] \cap C^{2}\left[J^{\prime}, R\right]$.

Proof By Lemma 1 and Lemma 2, we need only to show that the integral equation (2) has a solution $v \in L^{p}[J, R]$. The integral equation (2) can be written in the form

$$
v=G g v
$$

where $G$ is the linear integral operator defined by

$$
(G v)(t)=\int_{0}^{1} G(t, s) v(s) d s, \quad \forall t \in J
$$

and the nonlinear operator $g$ is defined by (18), which is bounded and continuous from $L^{p}[J, R]$ into $L^{q}[J, R]\left(\frac{1}{p}+\frac{1}{q}=1\right)$. It is well known that $G(t, s)$ is a $L^{2}$ positive-definite kernel with eigenvalues $\left\{\frac{1}{n^{2} \pi^{2}}\right\}(n=1,2,3, \ldots)$ and, by the continuity of $G(t, s)$, we have

$$
\int_{0}^{1} \int_{0}^{1}[G(t, s)]^{p} d s d t<\infty
$$

so $[22,23]$ the linear operator $G$ defined by $(20)$ is completely continuous from $L^{2}[J, R]$ into $L^{2}[, R]$ and also from $L^{q}[J, R]$ into $L^{p}[J, R]$, and $G=H H^{*}$, where $H=G^{\frac{1}{2}}$ (the positive square-root operator of $G$ ) is completely continuous from $L^{2}[J, R]$ into $L^{p}[J, R]$ and $H^{*}$ denotes the adjoint operator of $H$, which is completely continuous from $L^{q}[J, R]$ into $L^{2}[J, R]$. We now show that (19) has a solution $v \in L^{p}[J, R]$ is equivalent to the equation

$$
u=H^{*} g H u
$$

has a solution $u \in L^{2}[J, R]$. In fact, if $v \in L^{p}[J, R]$ is a solution of (19), i.e. $v=H H^{*} g v$, then $H^{*} g v=H^{*} g H H^{*} g v$, so, $u=H^{*} g v \in L^{2}[J, R]$ and $u$ is a solution of (22). Conversely, if $u \in$ $L^{2}[J, R]$ is a solution of (22), then $H u=H H^{*} g H u=G g H u$, so, $v=H u \in L^{p}[J, R]$ and $v$ is a 
solution of (19). Consequently, we need only to show that (22) has a solution $u \in L^{2}[J, R]$. It is well known $[22,23]$ that the functional $\Phi$ defined by

$$
\Phi(u)=\frac{1}{2}(u, u)-\int_{0}^{1} d t \int_{0}^{(H u)(t)} g(t, v) d v, \quad \forall u \in L^{2}[J, R]
$$

is a $C^{1}$ functional on $L^{2}[J, R]$ and its Fréchet derivative is

$$
\Phi^{\prime}(u)=u-H^{*} g H u, \quad \forall u \in L^{2}[J, R] .
$$

Hence we need only to show that there exists a $u \in L^{2}[J, R]$ such that $\Phi^{\prime}(u)=\theta(\theta$ denotes the zero element of $\left.L^{2}[, R]\right)$, i.e. $u$ is a critical point of functional $\Phi$.

By (4), (5), (16), and condition $\left(\mathrm{H}_{1}\right)$, we have

$$
\int_{0}^{u} g(t, v) d v=\int_{0}^{u+a(t)-a(1) t} f(t, w) d w-\int_{0}^{a(t)-a(1) t} f(t, w) d w, \quad \forall t \in J, u \in R
$$

and

$$
\begin{aligned}
\left|\int_{0}^{a(t)-a(1) t} f(t, w) d w\right| & \leq|a(t)-a(1) t|\left(a+b|a(t)-a(1) t|^{p-1}\right) \\
& \leq 2 a_{0}\left(a+b 2^{p-1} a_{0}^{p-1}\right)=a_{2}, \quad \forall t \in J .
\end{aligned}
$$

So, (25), (26), and condition $\left(\mathrm{H}_{2}\right)$ imply

$$
\begin{aligned}
\int_{0}^{(H u)(t)} g(t, v) d v & \leq \int_{0}^{(H u)(t)+a(t)-a(1) t} f(t, w) d w+a_{2} \\
& \leq c\{(H u)(t)+a(t)-a(1) t\}^{2}+d+a_{2} \\
& \leq 2 c\left\{[(H u)(t)]^{2}+[a(t)-a(1) t]^{2}\right\}+d+a_{2} \\
& \leq 2 c[(H u)(t)]^{2}+8 c a_{0}^{2}+d+a_{2}, \quad \forall u \in L^{2}[, R], t \in J .
\end{aligned}
$$

It is well known [24],

$$
\|G\|=\lambda_{1}=\frac{1}{\pi^{2}}
$$

where $G$ is defined by (20) and is regarded as a positive-definite operator from $L^{2}[J, R]$ into $L^{2}[J, R]$, and $\lambda_{1}$ denotes the largest eigenvalue of $G$. It follows from (23), (27), and (28) that

$$
\begin{aligned}
\Phi(u) & \geq \frac{1}{2}(u, u)-2 c(H u, H u)-8 c a_{0}^{2}-d-a_{2} \\
& =\frac{1}{2}(u, u)-2 c(G u, u)-8 c a_{0}^{2}-d-a_{2} \geq \frac{1}{2}(u, u)-\frac{2 c}{\pi^{2}}(u, u)-8 c a_{0}^{2}-d-a_{2} \\
& =\left(\frac{1}{2}-\frac{2 c}{\pi^{2}}\right)\|u\|^{2}-8 c a_{0}^{2}-d-a_{2}, \quad \forall u \in L^{2}[J, R],
\end{aligned}
$$

which implies by virtue of $0<c<\frac{\pi^{2}}{4}$ (see condition $\left.\left(\mathrm{H}_{2}\right)\right)$ that

$$
\lim _{\|u\| \rightarrow \infty} \Phi(u)=\infty
$$


So, there exists a $r>0$ such that

$$
\Phi(u)>\Phi(\theta)=0, \quad \forall u \in L^{2}[J, R],\|u\|>r .
$$

It is well known $[22,23]$ that the ball $T(\theta, r)=\left\{u \in L^{2}[J, R]:\|u\| \leq r\right\}$ is weakly closed and weakly compact and the functional $\Phi(u)$ is weakly lower semicontinuous, so, there exists $u^{*} \in T(\theta, r)$ such that

$$
\Phi\left(u^{*}\right)=\inf _{u \in T(\theta, r)} \Phi(u) \leq \Phi(\theta) .
$$

It follows from (31) and (32) that

$$
\Phi\left(u^{*}\right)=\inf _{u \in L^{2}[, R]} \Phi(u) .
$$

Hence $\Phi^{\prime}\left(u^{*}\right)=\theta$ and the theorem is proved.

Example 1 Consider the BVP

$$
\left\{\begin{array}{l}
-u^{\prime \prime}(t)=\frac{9}{2} u(t) \sin (t-u(t))-t^{3}, \quad \forall t \in J^{\prime}, \\
\left.\Delta u\right|_{t=t_{k}}=c_{k} \quad(k=1,2, \ldots, m), \\
\left.\Delta u^{\prime}\right|_{t=t_{k}}=d_{k} \quad(k=1,2, \ldots, m), \\
u(0)=u(1)=0
\end{array}\right.
$$

where $J=[0,1], 0<t_{1}<\cdots<t_{k}<\cdots<t_{m}<1, J^{\prime}=J \backslash\left\{t_{1}, \ldots, t_{k}, \ldots, t_{m}\right\}, c_{k}$ and $d_{k}(k=$ $1,2, \ldots, m)$ are any real numbers.

Conclusion BVP (33) has at least one solution $u \in P C^{1}[J, R] \cap C^{2}\left[J^{\prime}, R\right]$.

Proof Evidently, (33) is a BVP of the form (1) with

$$
f(t, u)=\frac{9}{2} u \sin (t-u)-t^{3} .
$$

It is clear that $f \in C[J \times R, R]$. By (34), we have

$$
|f(t, u)| \leq \frac{9}{2}|u|+1, \quad \forall t \in J, u \in R .
$$

Moreover, it is well known that

$$
|u| \leq \frac{1}{2}\left(1+u^{2}\right), \quad \forall u \in R .
$$

So, (35) and (36) imply that

$$
|f(t, u)| \leq \frac{9}{4} u^{2}+\frac{13}{4}, \quad \forall t \in J, u \in R,
$$

and consequently, condition $\left(\mathrm{H}_{1}\right)$ is satisfied for $p=3, a=\frac{13}{4}$ and $b=\frac{9}{4}$. On the other hand, choose $\epsilon_{0}$ such that

$$
0<\epsilon_{0}<\frac{1}{4}\left(\pi^{2}-9\right)
$$


For $|u| \geq \frac{1}{\epsilon_{0}}$, we have $|u| \leq \epsilon_{0} u^{2}$, so,

$$
|u| \leq \epsilon_{0} u^{2}+\frac{1}{\epsilon_{0}}, \quad \forall u \in R
$$

By (35), we have

$$
\int_{0}^{u} f(t, v) d v \leq \frac{9}{4} u^{2}+|u|, \quad \forall t \in J, u \in R .
$$

It follows from (38) and (39) that

$$
\int_{0}^{u} f(t, v) d v \leq\left(\frac{9}{4}+\epsilon_{0}\right) u^{2}+\frac{1}{\epsilon_{0}}, \quad \forall t \in J, u \in R .
$$

Since, by virtue of (37),

$$
0<\frac{9}{4}+\epsilon_{0}<\frac{\pi^{2}}{4}
$$

we see that (40) implies that condition $\left(\mathrm{H}_{2}\right)$ is satisfied for $c=\frac{9}{4}+\epsilon_{0}$ and $d=\frac{1}{\epsilon_{0}}$. Hence, our conclusion follows from Theorem 1.

By using the Mountain Pass Lemma and the Minimax Principle established by Ambrosetti and Rabinowitz [25, 26], we have obtained in [23] the existence of a nontrivial solution and the existence of infinitely many nontrivial solutions for a class of nonlinear integral equations. Since (2) is a special case of such nonlinear integral equations, we get the following result for (2).

Lemma 3 (Special case of Theorem 1 and Theorem 2 in [23]) Suppose the following.

(a) There exist $p>2$ and $a>0, b>0$ such that

$$
|g(t, v)| \leq a+b|v|^{p-1}, \quad \forall t \in J, v \in R .
$$

(b) There exist $0 \leq \tau<\frac{1}{2}$ and $M>0$ such that

$$
\int_{0}^{v} g(t, w) d w \leq \tau v g(t, v), \quad \forall t \in J,|v| \geq M
$$

(c) $\frac{g(t, v)}{v} \rightarrow 0$ as $v \rightarrow 0$ uniformly for $t \in J$ and $\frac{g(t, v)}{v} \rightarrow \infty$ as $|v| \rightarrow \infty$ uniformly for $t \in J$. Then the integral equation (2) has at least one nontrivial solution in $L^{p}[J, R]$. If, in addition,

(d) $g(t,-v)=-g(t, v), \forall t \in J, v \in R$.

Then the integral equation (2) has infinite many nontrivial solutions in $L^{p}[J, R]$.

Let us list more conditions for the function $f(t, u)$.

$\left(\mathrm{H}_{3}\right)$ There exist $0 \leq \tau<\frac{1}{2}$ and $M>0$ such that

$$
\int_{0}^{u} f(t, v+a(t)-a(1) t) d v \leq \tau u f(t, u+a(t)-a(1) t), \quad \forall t \in J,|u| \geq M
$$


$\left(\mathrm{H}_{4}\right) \frac{f(t, u+a(t)-a(1) t)}{u} \rightarrow 0$ as $u \rightarrow 0$ uniformly for $t \in J$, and $\frac{f(t, u+a(t)-a(1) t)}{u} \rightarrow \infty$ as $|u| \rightarrow \infty$ uniformly for $t \in J$.

$\left(\mathrm{H}_{5}\right) f(t,-u+a(t)-a(1) t)=-f(t, u+a(t)-a(1) t), \forall t \in J, u \in R$.

Theorem 2 Suppose that conditions $\left(\mathrm{H}_{1}\right),\left(\mathrm{H}_{3}\right)$, and $\left(\mathrm{H}_{4}\right)$ are satisfied. Then BVP $(1)$ has at least one solution $u \in P C^{1}[J, R] \cap C^{2}\left[J^{\prime}, R\right]$.If, in addition, condition $\left(\mathrm{H}_{5}\right)$ is satisfied, then $B V P(1)$ has infinitely many solutions $u_{n} \in P C^{1}[J, R] \cap C^{2}\left[J^{\prime}, R\right](n=1,2,3, \ldots)$.

Proof In the proof of Lemma 2, we see that condition $\left(\mathrm{H}_{1}\right)$ implies condition (a) of Lemma 3 (see (17)). On the other hand, it is clear that conditions $\left(\mathrm{H}_{3}\right),\left(\mathrm{H}_{4}\right),\left(\mathrm{H}_{5}\right)$ are the same as conditions (b), (c), (d) in Lemma 3, respectively. Hence the conclusion of Theorem 2 follows from Lemma 3, Lemma 2, and Lemma 1.

Example 2 Consider the BVP

$$
\left\{\begin{array}{l}
-u^{\prime \prime}(t)= \begin{cases}{[u(t)-t]^{3},} & \forall 0 \leq t<\frac{1}{2} \\
{[u(t)+3 t-3]^{3},} & \forall \frac{1}{2}<t \leq 1,\end{cases} \\
\left.\Delta u\right|_{t=\frac{1}{2}}=1, \\
\left.\Delta u^{\prime}\right|_{t=\frac{1}{2}}=-4 \\
u(0)=u(1)=0 .
\end{array}\right.
$$

Conclusion BVP (41) has infinite many solutions $u_{n} \in P C^{1}[J, R] \cap C^{2}\left[J^{\prime}, R\right](n=1,2,3, \ldots)$.

Proof Obviously, (41) is a BVP of form (1). In this situation, $J=[0,1], m=1, t_{1}=\frac{1}{2}$, $J^{\prime}=$ $[0,1] \backslash\left\{\frac{1}{2}\right\}, c_{1}=1, d_{1}=-4$, and

$$
f(t, u)= \begin{cases}(u-t)^{3}, & \forall 0 \leq t \leq \frac{1}{2} ; \\ (u+3 t-3)^{3}, & \forall \frac{1}{2}<t \leq 1 .\end{cases}
$$

It is clear that $f(t, u)$ is continuous on $J^{\prime} \times R$, left continuous at $t=t_{1}$, and the right limit $f\left(t_{1}^{+}, u\right)$ exists. By $(42)$, we have

$$
\begin{aligned}
|f(t, u)| & \leq\left(|u|+\frac{3}{2}\right)^{3} \leq\left(2 \max \left\{|u|, \frac{3}{2}\right\}\right)^{3} \\
& \leq 2^{3}\left(|u|^{3}+\left(\frac{3}{2}\right)^{3}\right)=8|u|^{3}+27, \quad \forall t \in J, u \in R,
\end{aligned}
$$

so, condition $\left(\mathrm{H}_{1}\right)$ is satisfied for $p=4, a=27$ and $b=8$. By (5), we have

$$
a(t)= \begin{cases}0, & \forall 0 \leq t \leq \frac{1}{2} \\ 3-4 t, & \forall \frac{1}{2}<t \leq 1\end{cases}
$$

so, $a(1)=-1$ and (42) and (43) imply

$$
f(t, u+a(t)-a(1) t)=u^{3}, \quad \forall t \in J, u \in R,
$$

and, consequently, $\left(\mathrm{H}_{3}\right)$ is satisfied for $\tau=\frac{1}{4}$ and any $M>0$. On the other hand, from (44) we see that conditions $\left(\mathrm{H}_{4}\right)$ and $\left(\mathrm{H}_{5}\right)$ are all satisfied. Hence, our conclusion follows from Theorem 2. 


\section{Competing interests}

The author declares that they have no competing interests.

\section{Acknowledgements}

Research was supported by the National Nature Science Foundation of China (No. 10671167).

Received: 16 December 2013 Accepted: 14 January 2014 Published: 07 Feb 2014

\section{References}

1. Lakshmikantham, V, Bainov, DD, Simeonov, PS: Theory of Impulsive Differential Equations. World Scientific, Singapore (1989)

2. Samoilenko, AM, Perestyuk, NA: Impulsive Differential Equations. World Scientific, Singapore (1995)

3. Benchohra, M, Henderson, J, Ntouyas, SK: Impulsive Differential Equations and Inclusions. Hindawi Publishing Corporation, New York (2006)

4. Agarwal, RP, O'Regan, D: Multiple nonnegative solutions for second order impulsive differential equations. Appl. Math. Comput. 114, 51-59 (2000)

5. Yan, B: Boundary value problems on the half line with impulses and infinite delay. J. Math. Anal. Appl. 259, 94-114 (2001)

6. Agarwal, RP, O'Regan, D: A multiplicity result for second order impulsive differential equations via the Leggett Williams fixed point theorem. Appl. Math. Comput. 161, 433-439 (2005)

7. Kaufmann, ER, Kosmatov, N, Raffoul, YN: A second-order boundary value problem with impulsive effects on an unbounded domain. Nonlinear Anal. 69, 2924-2929 (2008)

8. Guo, D: Positive solutions of an infinite boundary value problem for $n$ th-order nonlinear impulsive singular integro-differential equations in Banach spaces. Nonlinear Anal. 70, 2078-2090 (2009)

9. Guo, D: Multiple positive solutions for first order impulsive superlinear integro-differential equations on the half line. Acta Math. Sci. Ser. B 31(3), 1167-1178 (2011)

10. Guo, D, Liu, X: Multiple positive solutions of boundary value problems for impulsive differential equations. Nonlinear Anal. 25, 327-337 (1995)

11. Guo, D: Multiple positive solutions for first order nonlinear impulsive integro-differential equations in a Banach space Appl. Math. Comput. 143, 233-249 (2003)

12. Guo, D: Multiple positive solutions of a boundary value problem for $n$ th-order impulsive integro-differential equations in Banach spaces. Nonlinear Anal. 63, 618-641 (2005)

13. Xu, X, Wang, B, O'Regan, D: Multiple solutions for sub-linear impulsive three-point boundary value problems. Appl. Anal. 87, 1053-1066 (2008)

14. Jankowski, J: Existence of positive solutions to second order four-point impulsive differential problems with deviating arguments. Comput. Math. Appl. 58, 805-817 (2009)

15. Liu, Y, O'Regan, D: Multiplicity results using bifurcation techniques for a class of boundary value problems of impulsive differential equations. Commun. Nonlinear Sci. Numer. Simul. 16, 1769-1775 (2011)

16. Tian, $Y$, Ge, W: Applications of variational methods to boundary value problem for impulsive differential equations. Proc. Edinb. Math. Soc. 51, 509-527 (2008)

17. Nieto, JJ, O'Regan, D: Variational approach to impulsive differential equations. Nonlinear Anal., Real World Appl. 10, 680-690 (2009)

18. Zhang, Z, Yuan, R: An application of variational methods to Dirichlet boundary value problem with impulses. Nonlinear Anal., Real World Appl. 11, 155-162 (2010)

19. Chen, H, Sun, J: An application of variational method to second-order impulsive differential equations on the half line. Appl. Math. Comput. 217, 1863-1869 (2010)

20. Bai, L, Dai, B: Existence and multiplicity of solutions for an impulsive boundary value problem with a parameter via critical point theory. Math. Comput. Model. 53, 1844-1855 (2011)

21. Guo, D: A class of second-order impulsive integro-differential equations on unbounded domain in a Banach space. Appl. Math. Comput. 125, 59-77 (2002)

22. Krasnoselskii, MA: Topological Methods in the Theory of Nonlinear Integral Equations. Pergamon, Oxford (1964)

23. Guo, D: The number of nontrivial solutions to Hammerstein nonlinear integral equations. Chin. Ann. Math., Ser. B 7(2), 191-204 (1986)

24. Zaanen, AC: Linear Analysis. Interscience, New York (1958)

25. Ambrosetti, A, Rabinowitz, PH: Dual variational method in critical point theory and applications. J. Funct. Anal. 14, 349-381 (1973)

26. Rabinowitz, PH: Variational methods for nonlinear eigenvalue problems. In: Course of Lectures - CIME, Varenna, Italy (1974)

10.1186/1687-2770-2014-37

Cite this article as: Guo: Variational approach to a class of impulsive differential equations. Boundary Value Problem 2014, 2014:37 